\author{
Military Technical College \\ Kobry El-Kobbah, \\ Cairo, Egypt.
}

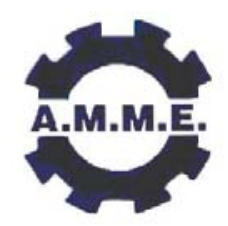
$13^{\text {th }}$ International Conference on Applied Mechanics and
Mechanical Engineering.

\title{
A POSTERIORI ERROR INDICATOR FOR COULOMB'S FRICTIONAL CONTACT
}

\author{
BECHEUR $^{*}$ A., TAHAKOURT ${ }^{* *}$ A. and COOREVITS ${ }^{* *} P$.
}

\begin{abstract}
In this paper, we present a new error indicator for the frictional contact problem governed by Coulomb's law. This indicator is built over the combination of two known methods. The first concerns the error obtained from the projection techniques where super convergence properties are used for smoothing the finite element stress fields. The second method takes into account the errors in the constitutive relation of the contact zones. By using both the error indicator and procedures of mesh adaptivities, we show example of optimised computations.
\end{abstract}

\section{KEY WORDS}

a posteriori error indicator, Coulomb's friction law, finite elements, smoothing stress fields, contact constitutive relations.

* Assistant Professor, Laboratoire de Technologie des Matériaux et de Génie des Procédés LTMGP. University of Bejaia. Route de Tharga-ouzemour.. 06000 BEJAIA. Algeria

** Professor, Laboratoire de Technologie des Matériaux et de Génie des Procédés LTMGP. University of Bejaia. Route de Tharga-ouzemour.. 06000 BEJAIA. Algeria

*** Professor, Laboratoire des Technologies Innovantes LTI, EA3899, Université de Picardie Jules Verne, France. 


\section{INTRODUCTION}

The numerical resolution of contact problems is often carried out by finite element methods. An important task consists in evaluating the numerical quality of the finite element computations by using either a priori or a posteriori error estimator. The a priori analysis gives information about convergence and rates of convergence of the solution. However, it does not allow us to quantify the descretisation errors. This quantification requires the definition of a posteriori error estimation.

For linear problems, several different approaches leading to various estimators have been developed. In particular, we cite the estimator introduced by Babuska and Rheinboldt [02] and based on the residual of the equilibrium equations; the estimators linked to the smoothing of finite element stresses performed by Zienkiewicz and Zhu [18]; and the estimator based on the errors in the constitutive relation developed by Ladeveze [12]. A review of different a posteriori estimators can be found in Zienkiewicz [19].

For non linear problems and especially for the non linearity of contact, the work available is much less abundant. However, an estimator based on the residuals has been developed by Wriggers et al [15]. By using a penalty method, they transformed a variational inequality into a variational equality. Nevertheless, this estimator explicitly uses a penalty parameter, which represents a major drawback. Moreover, for frictionless unilateral contact problems, there are principally three methods studied: the residual method by Carstensen et al [04] using a penalised approach; the study of error in the constitutive relations performed by Coorevits et al [06]; and the error indicator which uses the super convergence properties studied by Wriggers and Sherf [16].

In this paper, we are interested in the most used model of Coulomb's frictional law. In this domain, we can cite the works of Coorevits et al [07] and Louf et al [13], who used the estimator based on the error in the constitutive relation. This estimator requires the building of cinematically and statically admissible fields. The construction of these fields is complex and represents the principal difficulty for the use of this method. For the residual error estimator, it seems that only the contribution of Bostan and Han [03] for a bilateral contact problem is available. Although they used a Lagrangian approach, their studies were only limited to the Tresca's friction law. Indeed, so far, for the residual method applied to the Coulomb's law, the validation problem of the coefficients linked to different residual terms (such as interior and side residuals for each element) has remained unsolved. In the same article, a dependence of these coefficients to the mesh refinement and adaptation has also been shown. Consequently, the choice of these coefficients constitutes the principal difficulty for the use of this method.

The aim of this paper is to present a simple method that we have developed in Becheur et al [01] and where the performed error indicator would be easy to build and could take into account the error due to the contact. For this purpose, we propose to build this indicator by the combination of two known methods. The first method is based on the error obtained from the projection techniques where super convergence properties are used for smoothing the finite element stress fields. This method was developed by Zienkiewicz and Zhu [18]. The second method used by Coorevits et al [07] takes into account the error in the constitutive relation of the contact zones. The information given by this indicator is then coupled with mesh adaptivity techniques which provide the user 
with the desired quality and minimises the computational costs. The numerical implementations of the error indicator as well as optimised computations are performed.

\section{PROBLEM SETUP}

\section{General Notations}

We consider the two dimensional unilateral contact problem between two elastic bodies denoted by $\Omega^{1}$ and $\Omega^{2}$ (figure 01) respectively and $\Omega=\Omega^{1} \cup \Omega^{2}$. We assume that the boundary $\partial \Omega^{\ell}$ of $\Omega^{\ell}, \ell=1,2$ is divided into three parts:

$>\quad$ In the first part $\partial_{1} \Omega^{\ell}$, the displacement field is given by:

$$
\boldsymbol{U}_{\mid \partial_{1} \Omega^{\ell}}^{\ell}=\boldsymbol{U}_{\boldsymbol{d}}^{\ell}, \quad \ell=1,2 .
$$

To simplify, we suppose that:

$$
\boldsymbol{U}_{d}^{\sharp}=0, \quad \ell=1,2 .
$$

$>\quad$ In the second part $\partial_{2} \Omega^{\ell}$, surface forces of density $\boldsymbol{F}_{\boldsymbol{d}}^{\ell}$ are applied.

$>\quad$ The complementary part, denoted by $\partial_{C} \Omega^{\ell}$ such as $\partial_{C} \Omega^{\ell}=\partial \Omega^{\ell}-\left(\partial_{1} \Omega^{\ell} \cup \partial_{2} \Omega^{\ell}\right)$ is the candidate contact zone between the two solids. We suppose that $\partial_{C} \Omega^{1}=\partial_{C} \Omega^{2}$ which we denote by $\Gamma_{c}$.

Each body $\Omega^{\ell}$ is submitted to volume forces of density $\boldsymbol{f}_{d}^{\ell}$. We assume that the strain tensor $\varepsilon$ is linearised and we denote $\boldsymbol{K}^{\ell}$ by the elasticity operator associated with $\Omega^{\ell}$. The notation $\boldsymbol{n}^{\ell}$ stands for the unit outward normal on the boundary of $\Omega^{\ell}$.

\section{The Constitutive Relation of the Contact Zone}

In order to clearly express the contact error, we represent the contact zone $\Gamma_{\mathrm{c}}$ as a mechanical entity equipped with its constitutive relation. We choose the orientation of $\Gamma_{c}$ by setting $\mathbf{n}^{\mathrm{c}}=\mathbf{n}^{1}$.

Then we introduce on the interface $\Gamma_{c}$ the functions $\mathbf{W}^{\mathbf{1}}, \mathbf{W}^{\mathbf{2}}$, representing, two displacement fields on each side of the interface; and two fields of surface forces of densities $\mathbf{R}^{1}$ and $\mathbf{R}^{2}$ (stresses transmitted to $\Omega^{1}$ and $\Omega^{2}$ ) and an interior field of surface forces of density $\mathbf{R}^{\mathbf{C}}$.

The equilibrium of the interface is represented by

$$
R^{C}=R^{1} \text { and } R^{C}=-R^{2} \text { on } \Gamma \mathrm{C}
$$

such as:

$$
\boldsymbol{R}^{\ell}=\sigma^{\ell}: \boldsymbol{n}^{\ell}, \quad \ell=1,2
$$

Let us define $\mathbf{W}^{\mathbf{C}}$ the jump in displacement which, for the interface, plays a similar role as a strain:

$$
W^{c}=W^{1}-W^{2}
$$

For any vector $\mathbf{Z}$, we can write: $Z_{n}=\mathbf{Z}^{T} n^{c}$ and $\mathbf{Z}_{t}=\mathbf{Z}-\mathbf{Z}_{n} n^{c}$

The notation $\mathrm{T}$ represents the transposition. 
The relative displacement field $\mathbf{W}^{\mathrm{c}}=\mathrm{W}_{\mathrm{n}}{ }^{\mathrm{C}} \mathbf{n}^{\mathrm{c}}+\mathrm{W}_{\mathrm{t}}^{\mathrm{C}} \mathbf{t}^{\mathrm{c}}$ and the reaction density $\boldsymbol{R}^{C}=R_{n}^{C} \boldsymbol{n}^{C}+R_{t}^{C} \boldsymbol{t}^{C}$, verify the Coulomb's frictional contact at the interface $\Gamma_{\mathrm{c}}$.

Thus, the contact constitutive relations with Coulomb's law can be written as follows:

$$
\begin{aligned}
& W_{n}^{C}=W_{n}^{l}-W_{n}^{2} \leq 0 \\
& R_{n}^{C} \leq 0 \\
& R_{n}^{C} W_{n}^{C}=0 \\
& \left|R_{t}^{C}\right| \leq \mu\left|R_{n}^{C}\right| \\
& \text { if }\left|R_{t}^{C}\right|<\mu\left|R_{n}^{C}\right| \Rightarrow W_{t}^{C}=0 \\
& \text { if }\left|R_{t}^{C}\right|=\mu\left|R_{n}^{C}\right| \Rightarrow \exists \lambda \geq 0 \text { such as } W_{t}^{C}=-\lambda R_{t}^{C}
\end{aligned}
$$

The friction coefficient $\mu$ is assumed to be constant and positive on $\Gamma \mathrm{c}$.

The inequality in (2-6) expresses the non penetration of the two bodies; only contact or separation is allowed. The inequality in (2-7) states the sign condition on the normal reaction density and (2-8) represents the complementary condition.

We introduce the conjugate convex potentials $\varphi$ and $\varphi^{*}$ (Ekeland and Temam [10]):

$$
\begin{aligned}
& \varphi(\boldsymbol{V})=\left\{\begin{array}{ccc}
0 & \text { if } & V_{n} \geq 0 \\
+\infty & \text { if } & V_{n}<0
\end{array}\right.
\end{aligned}
$$

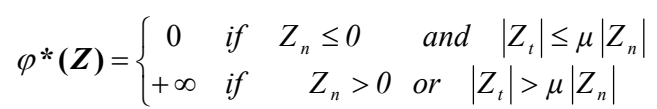

By following the scheme described in De Saxcé (1992), we introduce the function

$$
\Phi(\boldsymbol{V}, \boldsymbol{Z})=\varphi(\boldsymbol{V})+\varphi^{*}(\boldsymbol{Z})+\mu\left|Z_{n}\right|\left|V_{t}\right|
$$

A detailed proof of the properties of $\Phi(\boldsymbol{V}, \boldsymbol{Z})$ can be found in the previous reference. In particular, $\Phi(V, Z)$ is a bipotential, such as:

- For a fixed $\mathrm{V}, \Phi(\boldsymbol{V}, \boldsymbol{Z})$ is convex with respect to $\mathbf{Z}$

- For a fixed $Z, \Phi(\boldsymbol{V}, \boldsymbol{Z})$ is convex with respect to $\mathbf{V}$

- For any $\mathbf{V}$ and for any $\mathbf{Z}$, we have : $\quad \Phi(\boldsymbol{V}, \boldsymbol{Z})-\boldsymbol{Z}^{T} \boldsymbol{V} \geq 0$

On the other hand, the contact constitutive relations $(2-6)$ to $(2-11)$ can be written in the three following equivalent forms:

$$
\left\{\begin{array}{l}
-\boldsymbol{W}^{c} \in \partial \varphi^{*}\left(\boldsymbol{R}^{c}\right), \\
\boldsymbol{R}^{c} \in \partial \varphi\left(-\boldsymbol{W}^{c}\right), \\
\varphi\left(-\boldsymbol{W}^{c}\right)+\varphi^{*}\left(\boldsymbol{R}^{c}\right)+\mu\left|R_{n}^{c} \| W_{t}^{c}\right|+R_{t}^{c} W_{t}^{c}+R_{n}^{c} W_{n}^{c}=0
\end{array}\right.
$$

According to the definition introduced in (2-13a), we can write :

$$
\left\{\begin{array}{l}
-\boldsymbol{W}^{c} \in \partial \varphi^{*}\left(\boldsymbol{R}^{c}\right), \\
\boldsymbol{R}^{c} \in \partial \varphi\left(-\boldsymbol{W}^{c}\right), \\
\Phi\left(-\boldsymbol{W}^{c}, \boldsymbol{R}^{c}\right)+\boldsymbol{W}^{c^{r}} \boldsymbol{R}^{c}=0
\end{array}\right.
$$




\section{The Reference Problem}

The problem of unilateral contact of two elastic bodies with friction can be formulated as follows. Find the pair $\left(\left(\sigma^{\ell}, \boldsymbol{R}^{\ell}\right) ;\left(\mathrm{U}^{\ell}, \mathrm{W}^{\ell}\right)\right)$ defined in $\Omega^{\ell}, \ell=1,2$ and $\Gamma_{\mathrm{c}}$ such it satisfies:

$>$ The cinematic conditions:

$$
\boldsymbol{U}^{\ell}=0 \text { on } \partial_{1} \boldsymbol{\Omega}^{\ell} \quad \text { and } \quad \boldsymbol{U}^{\ell}=\boldsymbol{W}^{\ell} \text { on } \Gamma_{\mathrm{C}}
$$

$>$ The equilibrium equations :

$$
-\int_{\Omega^{\ell}} \boldsymbol{T} \boldsymbol{r}\left[\sigma^{\ell} \varepsilon\left(\boldsymbol{V}^{\ell}\right)\right] d \boldsymbol{d}+\int_{\Omega^{\ell}} \boldsymbol{f}_{d}^{\ell^{T}} \boldsymbol{V}^{\ell} d \boldsymbol{\Omega}+\int_{\partial_{2} \Omega^{\ell}} \boldsymbol{F}_{d}^{\ell^{T}} \boldsymbol{V}^{\ell} d \Gamma+\int_{\Gamma_{C}} \boldsymbol{R}^{\ell^{T}} \boldsymbol{V}^{\ell} d \Gamma=0 \quad \forall \boldsymbol{V}^{\ell} \in \boldsymbol{U}_{0}^{\ell}
$$

with $U_{0}^{\ell}=\left\{U^{\ell} \quad\right.$ defined and regular enough on $\Omega^{\ell}$ such as $U^{\ell}=0$ on $\left.\partial_{1} \Omega^{\ell}\right\}$ and $\mathbf{R}^{\mathbf{C}}$ $=\mathbf{R}^{1}$ and $\mathbf{R}^{\mathbf{C}}=-\mathbf{R}^{2}$ on $\Gamma_{\mathbf{C}}$

$>$ The constitutive relations:

$$
\left\{\begin{array}{l}
\sigma^{\ell}=\boldsymbol{K}^{\ell} \varepsilon\left(\boldsymbol{U}^{\ell}\right) \quad \ell=1,2 \\
\Phi\left(-\boldsymbol{W}^{c}, \boldsymbol{R}^{c}\right)+\boldsymbol{W}^{c^{r}} \boldsymbol{R}^{c}=0
\end{array}\right.
$$

The first existence result for problem (2-15) to (2-17) has been obtained by Necas et al [14] and Jarusek [11]. An improvement in Eck and Jarusek [09] states existence when the friction coefficient $\mu$ is lower than $\frac{\sqrt{3-4 v}}{2-2 v}, v$ denoting Poisson ratio in $\Omega$ such as $0<v<0.5$. About the uniqueness of the solution of this problem, there is to our knowledge no available result in the literature. Nevertheless, let us mention that there exist several laws "mollifying" Coulomb's frictional contact model and that such regularizations lead to more existence and uniqueness properties. Indeed, there are some uniqueness results given for the case of small coefficient of friction and with some kind of mollification (Duvaut [08]). However, for the more general problem without regularization, no uniqueness results are known. A review of mathematical results of existence and uniqueness of solutions to problems involving friction can be found in Andersson and Klarbring [01]. For the experimental results, it seems that the works available are much less abundant.

\section{THE ERROR INDICATOR FOR COULOMB'S FRICTIONAL CONTACT PROBLEM}

\section{The Finite Element Resolution}

The reference problem is usually resolved by the finite element method in displacements. It consists in finding the fields $\boldsymbol{U}_{h}{ }^{\ell}$ defined in $\Omega^{\ell} \quad, \ell=1,2$ of F.E type and verifying (2-1) such as:

$\forall \boldsymbol{V}_{h}^{\ell}$ of F.E type and such as $\boldsymbol{V}_{h}^{\ell}=0$ on $\partial_{1} \Omega^{\ell}$ and $\boldsymbol{V}_{h}^{\ell}=\boldsymbol{W}_{h}^{\ell}$ on $\Gamma_{\mathrm{C}}$

$>$ The equilibrium equations :

$$
-\int_{\Omega^{\prime}} \operatorname{Tr}\left[\varepsilon\left(\boldsymbol{U}_{h}^{\ell}\right) \boldsymbol{K} \varepsilon\left(\boldsymbol{V}_{h}^{\ell}\right)\right] d \Omega+\int_{\Omega^{\ell}} \boldsymbol{f}_{d}^{\ell^{T}} \boldsymbol{V}_{h}^{\ell} d \Omega+\int_{\partial_{2} \Omega^{\ell}} \boldsymbol{F}_{d}^{\ell^{T}} \boldsymbol{V}_{h}^{\ell} d \Gamma+\int_{\Gamma_{C}} \boldsymbol{R}^{\ell^{T} T} \boldsymbol{V}_{h}^{\ell} d \Gamma=0 \quad \forall \boldsymbol{V}_{h}^{\ell} \in \boldsymbol{U}_{0}^{\ell}
$$


with $U_{0}^{\ell}=\left\{U^{\ell} \quad\right.$ defined and regular enough on $\Omega^{\ell}$ such as $U^{\ell}=0$ on $\left.\partial_{1} \Omega^{\ell}\right\}$

$>\quad$ and the approximated stress field is calculated via the constitutive relation:

$$
\sigma_{\boldsymbol{h}}^{\ell}=\boldsymbol{K}^{\ell} \varepsilon\left(\boldsymbol{U}_{\boldsymbol{h}}^{\ell}\right) \quad \ell=1,2
$$

We consider the pair $\left(\left(\sigma^{\ell}, \boldsymbol{R}^{\ell}\right) ;\left(\mathrm{U}^{\ell}, \mathrm{W}^{\ell}\right)\right)$ with $, \ell=1,2$, the exact solution of the reference problem (2-15) to (2-17), and the pair $\left(\left(\sigma_{h}^{\ell}, \boldsymbol{R}_{h}^{\ell}\right) ;\left(\boldsymbol{U}_{h}^{\ell}, \boldsymbol{W}_{h}^{\ell}\right)\right)$ avec, $\ell=1,2$, the finite element solution of the approximated problem (3-1) to (3-3). If we compare the reference and the approximated problems, we can observe that both the finite element solution and the exact solution verify the cinematic condition (2-1) and the first equation of the constitutive relation (2-17a). But the approximated stress field $\sigma_{h}^{\ell}$ doesn't verify the equilibrium equation (2-16): in the displacement finite element method. The approximation is taken over the equilibrium equations.

Moreover, the fields $\boldsymbol{W}_{h}{ }^{C}$ and $\boldsymbol{R}_{h}{ }^{C}$ don't verify strictly the constitutive relations (2-14) on the contact zone. Thus, the quantity $\left(\Phi\left(-W_{h}{ }^{C}, R_{h}{ }^{C}\right)+W_{h}{ }^{C^{T}} \boldsymbol{R}_{h}{ }^{C}\right)$ is not equal to zero since $\boldsymbol{W}_{h}{ }^{C} \neq \boldsymbol{W}^{C}$ and $\boldsymbol{R}_{h}{ }^{C} \neq \boldsymbol{R}^{C}$. However, the function $\Phi\left(-\boldsymbol{W}_{h}^{C}, \boldsymbol{R}_{h}^{C}\right)$ must have a finite value. For this purpose, the reaction densities must always be included (corrected) in the friction Coulomb's cone and the non penetration condition satisfied. This imposes after a first resolution with the finite element method, an eventual correction of the reaction densities and displacements. Consequently, the condition of equilibrium will impose secondly the correction of the approximated stress fields $\sigma_{h}^{\ell}$.

\section{The Exact Definition of the Contact Error}

For all pairs $\left(\left(\sigma^{\ell}, \boldsymbol{R}^{\ell}\right) ;\left(\boldsymbol{U}^{\ell}, \boldsymbol{W}^{\ell}\right)\right)$ and $\left(\left(\sigma_{h}^{\ell}, \boldsymbol{R}_{h}^{\ell}\right) ;\left(\boldsymbol{U}_{h}^{\ell}, \boldsymbol{W}_{h}^{\ell}\right)\right)$ defined on $\Omega^{\ell}, \ell=1,2$, respectively the exact solution of the reference problem and the finite element solution of the approximated problem, lets set the quantity :

$$
\begin{aligned}
\boldsymbol{e}= & {\left[\sum_{\ell=1}^{2}\left\|\sigma^{\ell}-\sigma_{h}^{\ell}\right\|_{\sigma, \Omega^{\ell}}^{2}+2 \int_{\Gamma_{C}}\left(\Phi\left(-\boldsymbol{W}_{\boldsymbol{h}}^{\boldsymbol{C}}, \boldsymbol{R}_{\boldsymbol{h}}^{\boldsymbol{C}}\right)+\boldsymbol{W}_{\boldsymbol{h}}^{\boldsymbol{C}^{T}} \boldsymbol{R}_{\boldsymbol{h}}{ }^{C}\right) d \Gamma_{\boldsymbol{C}}\right]^{1 / 2} } \\
& \text { where }\left\|\sigma^{\ell}-\sigma_{h}^{\ell}\right\|_{\sigma, \Omega^{\ell}}=\left(\int_{\Omega^{\ell}}\left(\sigma^{\ell}-\sigma_{h}^{\ell}\right)^{T} \boldsymbol{K}^{-1}\left(\sigma^{\ell}-\sigma_{h}^{\ell}\right) d \Omega\right)^{1 / 2}
\end{aligned}
$$

By definition, the quantity e represents the exact definition of the error associated to the solution pair $\left(\left(\sigma_{h}^{\ell}, \boldsymbol{R}_{h}^{\ell}\right) ;\left(\boldsymbol{U}_{h}^{\ell}, \boldsymbol{W}_{h}^{\ell}\right)\right), \quad \ell=1,2$.

\section{Property 01 :}

For any pair of finite element solution $\left(\left(\sigma_{h}^{\ell}, \boldsymbol{R}_{h}^{\ell}\right) ;\left(\boldsymbol{U}_{\boldsymbol{h}}^{\ell}, \boldsymbol{W}_{\boldsymbol{h}}^{\ell}\right)\right)$ defined on $\boldsymbol{\Omega}^{\ell}, \ell=1,2$, we have e equal to zero if and only if $\left(\left(\sigma_{h}^{\ell}, \boldsymbol{R}_{h}^{\ell}\right) ;\left(\boldsymbol{U}_{h}^{\ell}, \boldsymbol{W}_{h}^{\ell}\right)\right)$ is the exact solution of the reference problem. 
Indeed, if $\left(\left(\sigma_{h}^{\ell}, \boldsymbol{R}_{h}^{\ell}\right) ;\left(\boldsymbol{U}_{h}^{\ell}, \boldsymbol{W}_{h}^{\ell}\right)\right)$ is the exact solution, we will have $\sigma^{\ell}=\sigma_{h}^{\ell}, \ell=1,2 ;$ and $\left(\Phi\left(-W_{h}{ }^{C}, \boldsymbol{R}_{h}{ }^{C}\right)+\boldsymbol{W}_{h}{ }^{C^{T}} \boldsymbol{R}_{h}{ }^{C}\right)=0$ since $\boldsymbol{W}_{h}{ }^{C}$ and $\boldsymbol{R}_{h}{ }^{C}$ will verify the constitutive relations $(2.6)$ to $(2.11)$ in the contact zone.

\section{Property 02 :}

For any pair of finite element solution $\left(\left(\sigma_{h}^{\ell}, \boldsymbol{R}_{h}^{\ell}\right) ;\left(\boldsymbol{U}_{h}^{\ell}, \boldsymbol{W}_{h}^{\ell}\right)\right)$ defined on $\boldsymbol{\Omega}^{\ell}, \ell=1,2$, the defined error $\mathbf{e}$ is either positive or equal to zero.

Proof : According to (2-13a), we can write :

$\boldsymbol{e}=\left[\sum_{\ell=1}^{2}\left\|\sigma^{\ell}-\sigma_{h}^{\ell}\right\|_{\sigma, \Omega^{\ell}}^{2}+2 \int_{\Gamma_{C}}\left(\varphi\left(-\boldsymbol{W}_{h}{ }^{c}\right)+\varphi^{*}\left(\boldsymbol{R}_{h}{ }^{c}\right)+\mu\left|R_{h n}{ }^{C}\right|\left|W_{h t}^{C}\right|+R_{h t}^{C} W_{h t}^{C}+R_{h n}{ }^{C} W_{h n}^{C}\right) d \Gamma_{C}\right]^{1 / 2}$

Being the energy norm on the stress tensor fields, the first term $\left\|\sigma^{\ell}-\sigma_{h}^{\ell}\right\|_{\sigma, \Omega^{\ell}}^{2}$ is always positive or equal to zero. For the second term, we can remark that the integral above is always positive: it is equal to $+\infty$ if $-\boldsymbol{W}_{\boldsymbol{h}}^{\boldsymbol{C}} \notin \partial \varphi^{*}\left(\boldsymbol{R}_{\boldsymbol{h}}^{\boldsymbol{C}}\right)$ (if the non penetration condition is not satisfied) or if $\boldsymbol{R}_{\boldsymbol{h}}^{\boldsymbol{C}} \notin \partial \varphi\left(-\boldsymbol{W}_{\boldsymbol{h}}^{C}\right)$ (if the normal reaction density is positive or the tangential reaction density is outside the Coulomb's cone). Otherwise, it is positive because $R_{h_{n}}^{C} W_{h_{n}}^{C} \geq 0$ (according to the relations (2.6) and (2.7)) and $\mu\left|R_{h_{n}}^{C}\right|\left|W_{h_{t}}^{C}\right|+R_{h_{t}}^{C} W_{h_{t}}^{C} \geq 0$ (according to the relations $(2.9)$ to $(2.11)$ ) In deed,for the latest inequation, there exist two cases. In the first case, we may have $\left|R_{h t}^{C}\right|<\mu\left|R_{h n}^{C}\right|$. This implies according to the relation (2.10) that: $W_{h_{t}}^{C}=0$ and $\mu\left|R_{h_{n}}^{C}\right|\left|W_{h_{t}}^{C}\right|+R_{h_{t}}^{C} W_{h_{t}}^{C}=0$. In the second case, we may have $\left|R_{h t}^{C}\right|=\mu\left|R_{h n}^{C}\right|$. This implies according to the relation (2.11) that $W_{h_{t}}^{C} \neq 0$ and $R_{h_{t}}^{C} W_{h_{t}}^{C}=-\left|R_{h_{t}}^{C}\right|\left|W_{h_{t}}^{C}\right|$ so that $\mu\left|R_{h_{n}}^{C} \| W_{h_{t}}^{C}\right|+R_{h_{t}}^{C} W_{h_{t}}^{C}=0$. Moreover, according to the property due to De Saxcé (1992) and written in (2-13b), the term $\left(\Phi\left(-\boldsymbol{W}_{h}{ }^{C}, \boldsymbol{R}_{h}{ }^{C}\right)+\boldsymbol{W}_{h}{ }^{C^{T}} \boldsymbol{R}_{h}{ }^{C}\right)$ in (3.4) is always positive or equal to zero. This concludes the proof $\square$.

Moreover, the error quantity e must have a finite value. For this purpose, we must have:

$$
\varphi\left(-\boldsymbol{W}_{h}^{C}\right)=\varphi^{*}\left(\boldsymbol{R}_{h}^{C}\right)=0
$$

In other words, we must have $-\boldsymbol{W}_{\boldsymbol{h}}^{\boldsymbol{C}} \in \partial \varphi^{*}\left(\boldsymbol{R}_{\boldsymbol{h}}^{\boldsymbol{C}}\right)$ and $\boldsymbol{R}_{\boldsymbol{h}}^{\boldsymbol{C}} \in \partial \varphi\left(-\boldsymbol{W}_{\boldsymbol{h}}^{\boldsymbol{C}}\right)$. Nevertheless, this is not always verified by the finite element solutions. Consequently, the correction at the interface $\Gamma_{c}$ of the displacement fields $\boldsymbol{W}_{h}^{C}$ and reaction densities $\boldsymbol{R}_{h}^{C}$ is indispensable. And then, the condition of the equilibrium requires a second resolution we can name the corrected problem which consists of finding the fields $\boldsymbol{U}_{c}{ }^{\ell}$ defined in $\Omega^{\ell} \quad, \ell=1,2$ of F.E type and verifying (2-1) such as:

$>\quad \forall \boldsymbol{V}_{\boldsymbol{h}}^{\ell}$ of F.E type and such as $\boldsymbol{V}_{\boldsymbol{h}}^{\ell}=0$ on $\partial_{1} \Omega^{\ell}$ and $\boldsymbol{V}_{\boldsymbol{h}}^{\ell}=\boldsymbol{W}_{\boldsymbol{h}}^{\ell}$ on $\Gamma_{\mathrm{C}}$ 
$>\quad-\boldsymbol{W}_{c}^{C} \in \partial \varphi^{*}\left(\boldsymbol{R}_{h}^{C}\right)$ and $\boldsymbol{R}_{c}^{C} \in \partial \varphi\left(-\boldsymbol{W}_{h}^{C}\right)$

$>$ The equilibrium equations :

$$
-\int_{\Omega^{\ell}} \boldsymbol{T r}\left[\varepsilon\left(\boldsymbol{U}_{\boldsymbol{c}}^{\ell}\right) \boldsymbol{K} \varepsilon\left(\boldsymbol{V}_{\boldsymbol{h}}^{\ell}\right)\right] d \Omega+\int_{\Omega^{\ell}} \boldsymbol{f}_{d}^{\ell^{T}} \boldsymbol{V}_{h}^{\ell} d \Omega+\int_{\partial_{\Omega^{\ell}}} \boldsymbol{F}_{d}^{\ell^{T}} \boldsymbol{V}_{h}^{\ell} d \Gamma+\int_{\Gamma_{C}} \boldsymbol{R}_{c}^{\ell^{T}} \boldsymbol{V}_{h}^{\ell} d \Gamma=0 \quad \forall \boldsymbol{V}_{h}^{\ell} \in \boldsymbol{U}_{0}^{\ell}
$$

with $U_{0}^{\ell}=\left\{U^{\ell} \quad\right.$ defined and regular enough on $\Omega^{\ell}$ such as $U^{\ell}=0$ on $\left.\partial_{1} \Omega^{\ell}\right\}$

The corrected stress field is calculated via the constitutive relation: $\sigma_{c}^{\ell}=\boldsymbol{K}^{\ell} \varepsilon\left(\boldsymbol{U}_{c}^{\ell}\right) \quad \ell=1,2$ and the pair $\left(\left(\sigma_{c}^{\ell}, \boldsymbol{R}_{c}^{\ell}\right) ;\left(\boldsymbol{U}_{c}^{\ell}, \boldsymbol{W}_{c}^{\ell}\right)\right)$ is the corrected solution so that $\varphi\left(-\boldsymbol{W}_{c}^{C}\right)=\varphi^{*}\left(\boldsymbol{R}_{c}^{C}\right)=0$.

This lead to the final definition of the error which can be written as:

$$
\boldsymbol{e}=\left[\sum_{\ell=1}^{2}\left\|\sigma^{\ell}-\sigma_{c}^{\ell}\right\|_{\sigma, \Omega^{\ell}}^{2}+2 \int_{\Gamma_{C}}\left(\mu\left|R_{c n}^{C} \| W_{c t}^{C}\right|+R_{c t}^{C} W_{c t}^{C}+R_{c n}^{C} W_{c n}^{C}\right) d \Gamma_{C}\right]^{1 / 2}
$$

\section{The Error Indicator}

The exact solution is generally unknown; we must then calculate an indicator of the error over the finite element solution. The idea consists in substituting, the exact stress field $\sigma^{\ell}, \ell=1,2$ in (3-8) by a stress field $\tilde{\sigma}^{\ell}, \ell=1,2$ whose properties of internal equilibrium and continuity between elements are verified sufficiently.

Moreover, it is known that the finite element solution is not smooth and regular enough compared to the exact solution. Thus, Zienkiewicz and Zhu [18] had the idea of building from the finite element solution, a new stress field of high degree of smoothing and which is assumed to be as near as possible to the exact solution. For this purpose, they used super convergence properties of the stress fields. Indeed, Hinton and Campbell (1974) observed that the finite element solution oscillated over the exact solution and in some points, named super convergent points, the two solutions coincided.

Consequently, we can estimate the error by substituting in the first term of (3-8) $\left\|\sigma^{\ell}-\sigma_{c}^{\ell}\right\|_{\sigma, \Omega^{\ell}}$ by $\left\|\tilde{\sigma}^{\ell}-\sigma_{c}^{\ell}\right\|_{\sigma, \Omega^{\ell}}$ such as:

$$
\left\|\tilde{\sigma}^{\ell}-\sigma_{c}^{\ell}\right\|_{\sigma, \Omega^{\ell}}=\left(\int_{\Omega^{\ell}}\left(\tilde{\sigma}^{\ell}-\sigma_{c}^{\ell}\right)^{T} K^{-1}\left(\tilde{\sigma}^{\ell}-\sigma_{c}^{\ell}\right) d \Omega\right)^{1 / 2}
$$

Finally we can compute an approximation of the error denoted by $\mathbf{e}_{\mathbf{s}}$, so that:

$$
\boldsymbol{e}_{\boldsymbol{S}}=\left[\sum_{\ell=1}^{2}\left\|\widetilde{\sigma}^{\ell}-\sigma_{c}^{\ell}\right\|_{\sigma, \Omega^{\ell}}^{2}+2 \int_{\Gamma_{c}}\left(\mu\left|R_{c n}^{c}\right|\left|W_{c t}^{c}\right|+R_{c t}^{c} W_{c t}^{C}+R_{c n}^{c} W_{c n}^{C}\right) d \Gamma_{C}\right]^{1 / 2}
$$

Where $\tilde{\sigma}^{\ell}$ is the smoothed stress field over each body $\Omega^{\ell}, \ell=1,2$.

On the other hand, $\tilde{\sigma}^{\ell}$ can be obtained either by the classical global method projection also named $Z Z^{1}$ and developed by Zienkiewicz and Zhu [18], or by the superconvergent patch recovery (SPR) method by the same authors Zienkiewicz and Zhu [17]. The second method (SPR) is more accurate than the global method $Z Z^{1}$. Nevertheless, it 
seemed to be more difficult to implement and to control, particularly, in a finite element contact program. That is why, we have chosen, in this article, to study firstly the quality of use of the global method.

In this method, the smoothed stress field can be obtained by the minimisation in the sense of least squares, of the distances between the continued field $\tilde{\sigma}$ and the corrected finite element field $\sigma_{c}^{\ell}$. The nodal values $\left(\widetilde{\sigma}_{i}^{\ell}\right)$ of the smoothed field can be obtained by minimising the two following functions:

$$
\boldsymbol{F}_{\ell}\left(\tilde{\sigma}_{i}^{\ell}\right)=\int_{\Omega^{\prime}}\left(\tilde{\sigma}^{\ell}-\sigma_{c}^{\ell}\right)^{2} d \Omega, \quad \ell=1,2
$$

It results in two systems of linear independent equations:

$$
\frac{\partial \boldsymbol{F}_{\ell}}{\partial \widetilde{\boldsymbol{\sigma}}_{i}^{\ell}}=0 \Rightarrow \boldsymbol{A}^{\ell} \widetilde{\boldsymbol{\sigma}}^{\ell}=\boldsymbol{b}^{\ell}, \quad \ell=1,2 .
$$

Moreover, we associate to this error measure of descretisation the global relative error which is defined by:

$$
\varepsilon=\left[\frac{\sum_{\ell=1}^{2} \mid \widetilde{\sigma}^{\ell}-\sigma_{c}^{e} \|_{\sigma, \Omega^{\prime}}^{2}+2 \int_{\Gamma_{c}}\left(\mu||_{c n}^{c} \|\left|W_{c t}^{c}\right|+R_{c t}^{c} W_{c t}^{c}+R_{c n}^{c} W_{c n}^{c}\right) d \Gamma_{c}}{\sum_{\ell=1}^{2} \mid \widetilde{\sigma}^{\ell}+\sigma_{c}^{e} \|_{\sigma, \Omega^{\prime}}^{2}}\right]^{1 / 2}
$$

Thus $\varepsilon$ consists of a global accuracy which allows evaluating the global quality of the finite element solution. Let us consider $\mathrm{E}$ a part of $\boldsymbol{\Omega}^{\ell}$. Then, we define the local contribution of $\mathrm{E}$ to the global error $\varepsilon$ by the quantity $\varepsilon_{\mathrm{E}}$, such as:

$$
\varepsilon_{E}=\left[\frac{\left\|\widetilde{\sigma}^{\ell}-\sigma_{c}^{\ell}\right\|_{\sigma, E}^{2}+\int_{\Gamma_{c} \wedge E}\left(\mu\left|R_{c n}^{c} \| W_{c t}^{c}\right|+R_{c t}^{c} W_{c t}^{c}+R_{c n}^{c} W_{c n}^{c}\right) \mathrm{d} \boldsymbol{\Gamma}_{c}}{\sum_{\ell=1}^{2}\left\|\widetilde{\sigma}^{\ell}+\sigma_{c}^{\ell}\right\|_{\sigma, \Omega^{\ell}}^{2}}\right]^{1 / 2}
$$

In practical situations, $\mathrm{E}$ is an element of the mesh descretisation associated to $\Omega^{\ell}$. The local contributions allow obtaining information concerning the errors located on the structure. By construction, one has

$$
\varepsilon^{2}=\sum_{E} \varepsilon_{E}^{2}
$$

\section{NUMERICAL STUDIES}

The goal of a mesh adaptation procedure is to guarantee a certain level of precision to the finite element user, by minimising the computational costs. We will use the hgeneration, which is the most frequently used procedure: the size and topologies of the elements are changed while the type of finite element functions on the different meshes remains the same.

A mesh $\mathbf{T}^{*}$ is said to be optimal for an error measure $\varepsilon$ if: 


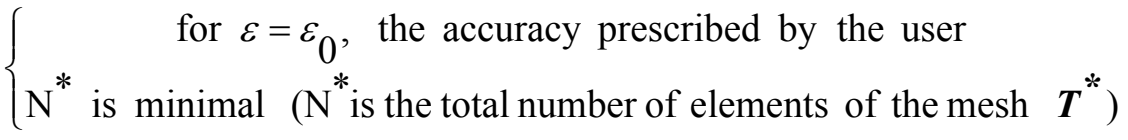

In order to solve the problem in (4-1), we adopt the following technique (Coorevits et al [05]):

- Computation on a coarse mesh $\mathbf{T}$

- Computation of both the global relative error $\varepsilon$ and the local contributions $\varepsilon_{E}$

- Determination of the optimised mesh $\mathbf{T}^{*}$

- And a second computation on the new mesh $\mathbf{T}^{*}$.

The implementation of this method is achieved using CASTEM 2000 developed at the CEA in France. We consider the problem of two elastic bodies initially in contact (figure 02). The upper body is submitted to a uniform load of $10 \mathrm{Nmm}^{-2}$. The body $\Omega^{2}$ is fixed on its lower side. The two materials are identical $(E=130 \mathrm{GPa}$ and $v=0.3)$. The dimensions are $100 \mathrm{~mm} \times 100 \mathrm{~mm}$ and $200 \mathrm{~mm} \times 200 \mathrm{~mm}$ and the friction coefficient is $\mu$ $=0.3$.

The initial mesh is made of 398 three nodes elements, and 370 nodes (figure 03). By applying the developed indicator, a global accuracy $\varepsilon$ of $6.58 \%$ is obtained. We show the deformed bodies in figure 04 and the contribution to the error $\varepsilon_{\mathrm{E}}$ in figure 05.

The contact pressures are drawn in figure 06. We can observe that the computed tangential pressure is outside the friction cone (on both extreme meshes) and it can be successfully modified to compute the error indicator.

A global accuracy $\varepsilon_{0}$ of $05 \%$ is prescribed. The resolution of the problem (4.1) gives an optimised mesh made of 426 three nodes elements, 259 nodes, and the developed indicator provides a global accuracy $\varepsilon$ of $5.32 \%$ (figure $07 a$ ).

By using the error in constitutive relation method, this example was also treated in Coorevits et al [07] (see figure 07b). In deed, for a same prescribed error of $05 \%$, we can observe on this figure, that after remeshing, a final error of $5.46 \%$ is obtained for an optimised mesh of 381 three nodes elements. Whereas the use of the developed method gives a final error of $\mathbf{5 . 3 2} \%$ for an optimised mesh of 426 three nodes elements (figure 07a). On the other hand, by comparing the mesh adaptation obtained by the two methods, we can observe on these figures (07a and 07b) that, despite its low cost, the developed method proves its efficiency by sufficiently taking into account the contact error and providing an optimised mesh on the contact zone for approximately the same level of accuracy.

\section{CONCLUSION}

After this study, we can conclude that the method developed presents the principal advantage of a low cost compared to other methods. This method has allowed overcoming the drawbacks related to the construction difficulties of the admissible fields, and the choice of coefficients for the residual method. Indeed, by combining the projection techniques and the error in the constitutive relation on the contact zone, we have developed a rather simple error indicator to build and to implement in a finite element code. It also gives large possibilities to be applied either on non linear 
behaviours or on three dimensional contact problems. Moreover, when comparing with the results obtained by the error in the constitutive relations, the numerical study has shown that this indicator presents the ability to take into account the contact error and to provide optimised meshes in the structure.

\section{REFERENCES}

[1] Becheur, A. Tahakourt, A. Coorevits, P. An a posteriori error indicator for Coulomb's frictional contact. To be published and available on line in Mechanics Research Communication Journal Elsevier Ed (2008).

[2] Babuska, I., Rheinboldt, W.C.,. Error estimates for adaptive finite element computations. SIAM J. Numer. Anal. vol. 15, n4, 736-754. (1978)

[3] Bostan, V., Han, W.,. A posteriori error analysis for FEM solutions of a frictional contact problem, Comput. Meth Appl. Mech. Engrg. Vol 195, pp 1252-1274, (2006).

[4] Carstensen, C., Sherf, O., and Wriggers, P., Adaptive finite elements for elastic bodies in contact, SIAM J. Sci. Comput. 20,. 1605-1626. (1999).

[5] Coorevits, P., Ladeveze, P., Pelle, J.P. Mesh optimization for problems with steep gradient areas. Engineering computations. 11, 129-144. (1994).

[6] Coorevits, P., Hild, P.,. Pelle, J.P. A posteriori error estimation for unilateral contact with matching and non matching meshes. Comp Meth. Appl. Mech. and Engrg. 186, 65-83, (2000).

[7] Coorevits, P., Hild, P., Hjiaj, M. A posteriori error control for finite element approximations of Coulomb frictional contact. SIAM J. Sci. Comp. vol 23, $\mathrm{n}^{\circ} 3$, 976-999. (2001)

[8] Duvaut, G., Lions, J.L., Les inéquations en mécanique et en physique, Dunod editions. Paris. (1980).

[9] Eck, C., Jarusek, J. Existence results for the static contact problem with coulomb friction. Math. Models Methods Appl. Sci.. 8,. 445-468 (1998).

[10] Ekeland, I., Temam, R. Convex Anal. Variational Problems; Amsterdam, NorthHolland (1976).

[11] Jarusek, J. Contact Problems with Bounded Friction. Czechoslovak. Math. J. 33, 237-261 (1983).

[12] Ladeveze, P. Comparaison de modèles de milieux continus, Ph.D. thesis, Paris VI, university (1975)

[13] Louf, F., Combe, J.P., Pelle, J.P. Constitutive error estimator for the control of contact problems involving friction. Computers and Structures J. 81, 1759-1772 (2003).

[14] Necas, J., Jarusek, J., Haslinger, J.,. On the solution of the variational inequality to the Signorini problem with small friction, Boll. Un. Mat. Ital. B (5), 17, . 796-811 (1980).

[15] Wriggers, P., Scherf, O., Carstensen, C. Adaptive techniques for the contact of elastic bodies. in Recent developments in FEM analysis, eds. Hughes, Onate, Zienkiewicz, 78-86. (1994)

[16] Wriggers, P., Scherf, O. Different A Posteriori Error Estimators And Indicators For Contact Problems. Math Comput Modelling Vol 28, No 4-8, 437-447, (1998). 
[17] Zienkiewicz, O. C., Zhu, J. Z.,. The Superconvergent Patch Recovery and adaptive finite element refinement. Comp Methods in Applied Mechanics and Engineering, Vol 101, Iss 1-3, 207-224 (1992).

[18] Zienkiewicz, O.C., Zhu, J.Z. A simple error estimator and adaptive procedure for practical engineering analysis. Int. J. for Num. Meth. in Engng. 24, 337357(1987).

[19] Zienkiewicz, O.C. The background of error estimation and adaptivity in finite element computations. Comput Methods Appl Mech Engrg. 195, 207-213 (2006).

Figures

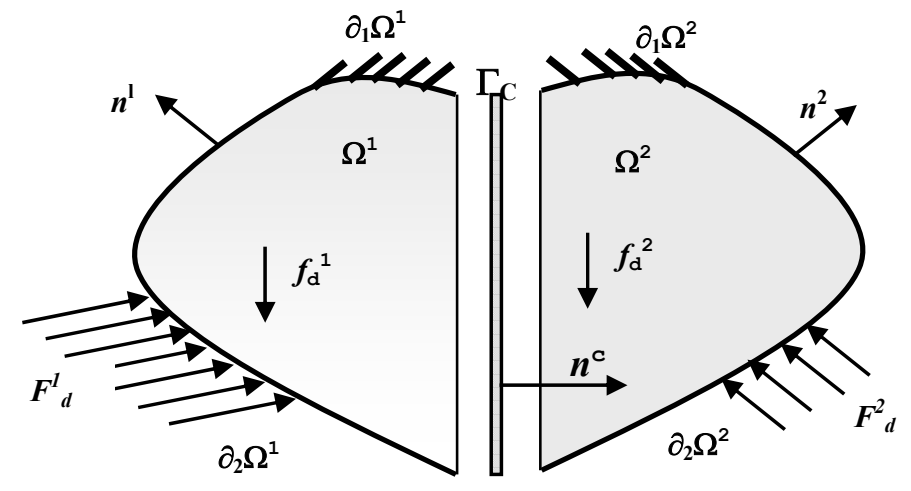

Figure 1 : Setting of the problem

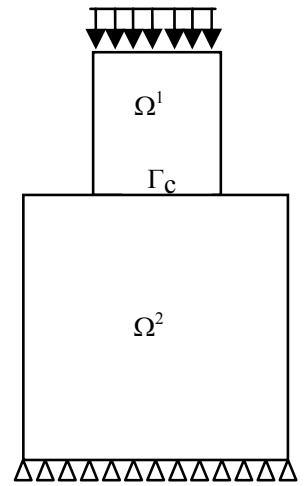

Figure 2 : Setting of the problem

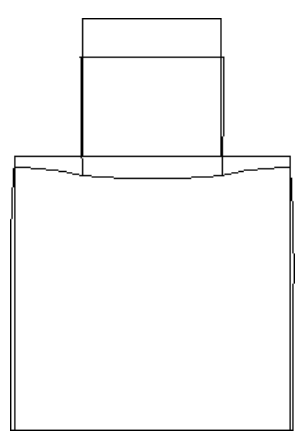

Figure 4: Deformed shapes

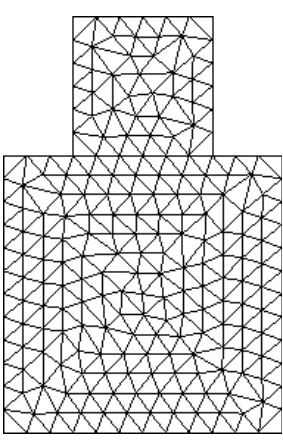

Figure 3 : Initial mesh : 398 three node elements, 370 nodes, $\varepsilon=6.58 \%$

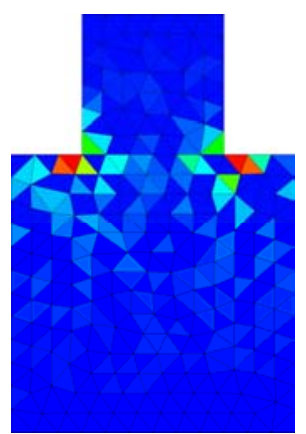

Figure 5: Local contributions $\varepsilon_{\mathrm{E}}$ 


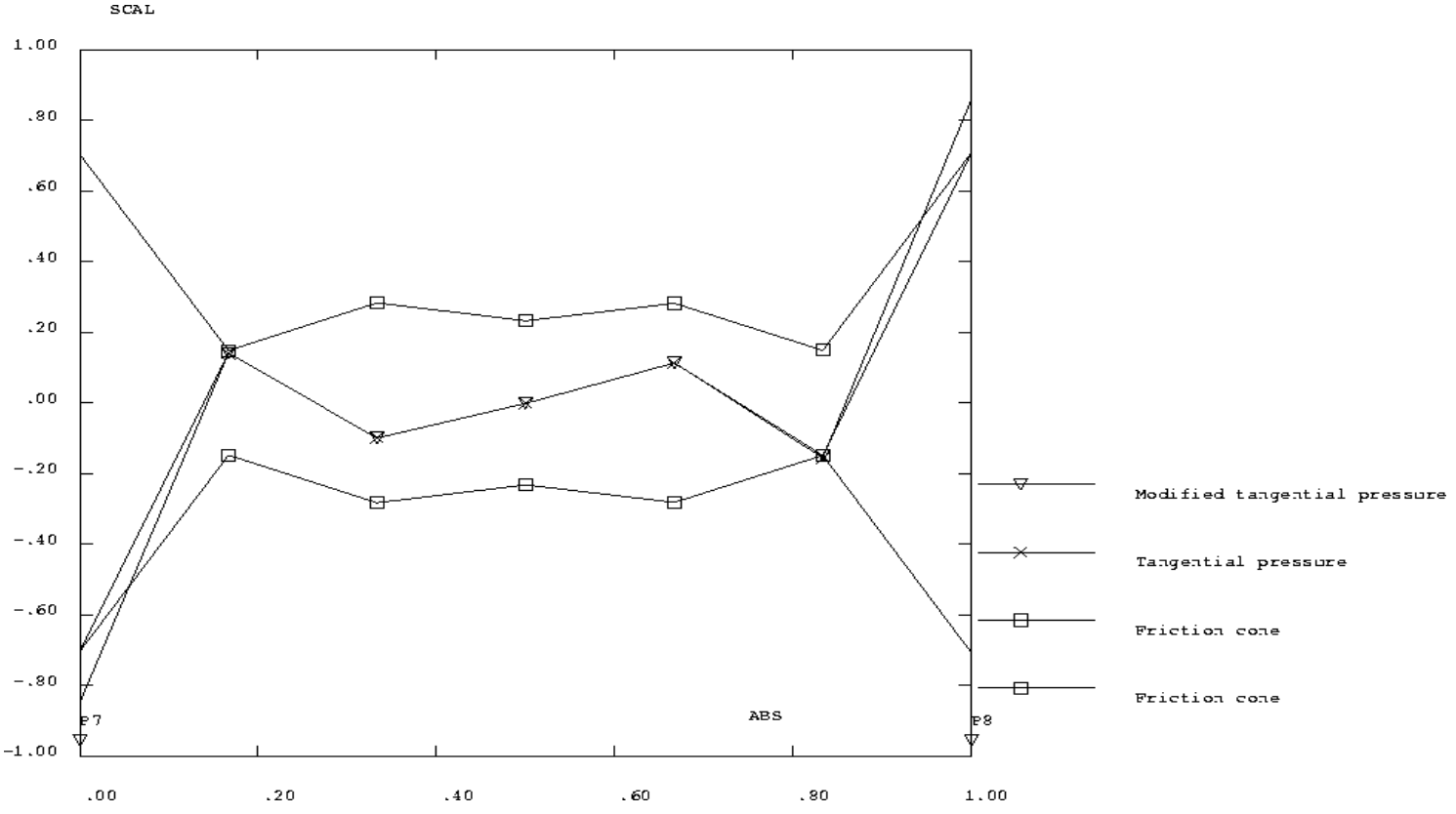

Figure 6: Contact pressures.

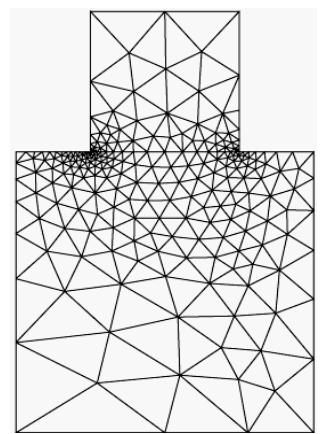

Figure 7a: optimised mesh of 426 three nodes elements, 259 nodes for a final accuracy of $5.32 \%$ (by using the developed method)

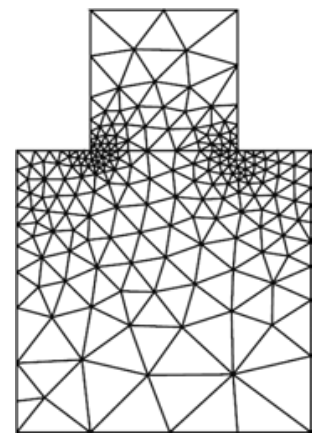

Figure 7b: optimised mesh of 381 three nodes elements, 376 nodes for a final accuracy of $5.46 \%$ (method developed by Coorevits et al 2001) 